\title{
Early marginal bone stability of dental implants placed in a transalveolarly augmented maxillary sinus: a controlled retrospective study of surface modification with calcium ions
}

\author{
Eduardo Anitua ${ }^{1,2,3,5^{*}}$ (D) Laura Piñas ${ }^{4}$ and Mohammad Hamdan Alkhraisat ${ }^{2,3}$
}

\begin{abstract}
Background: Recently, components of the extracellular cellular matrix have been assessed to enhance the biological response to dental implants. This study aims to assess the effect of surface modification with calcium ions on the early marginal bone loss of dental implants placed in a transalveolarly augmented maxillary sinus.

Methods: A retrospective study of transalveolar sinus floor augmentation was conducted in a single private dental clinic. The predictor variable was the surface of the dental implant. The primary outcome was the marginal bone loss. The secondary outcomes were the intraoperative complications and the dental implant failure. Descriptive analysis was performed for patients' demographic data and implant details.

Results: Fifty-one patients with a mean age of $58 \pm 11$ years had a mean follow-up time of 13 months. Thirty-four dental implants had a $\mathrm{Ca}^{2+}$-modified hydrophilic surface, and 31 had no $\mathrm{Ca}^{2}$ (control). The experimental group showed a statistically significant lower marginal bone loss ( $0.36 \pm 0.42$ vs $0.61 \pm 0.39 \mathrm{~mm})$. However, there were no statistically significant differences in the implant survival. No implant failed in the experimental group while two implants failed in the control group.
\end{abstract}

Conclusions: The modification of an acid-etched surface with calcium ions seems to reduce the marginal bone remodeling around the dental implants, placed after transalveolar sinus floor elevation.

Keywords: Calcium, Dental implant, Implant surface, Marginal bone loss, Osseointegration

\section{Background}

Dental implants are nowadays the treatment of choice to replace missing teeth due to their high predictability and long-term success [1]. This success is the outcome of several cellular and molecular events that take place at the implant-bone interface. Although the process of osseointegration is not fully understood, research is ongoing to enhance and accelerate this process. Moderately rough implant surface has enhanced implant

\footnotetext{
* Correspondence: eduardo@fundacioneduardoanitua.org

${ }^{1}$ Private practice in oral implantology, Clínica Eduardo Anitua, Vitoria, Spain ${ }^{2}$ University Institute for Regenerative Medicine and Oral Implantology - UIRMI (UPV/EHU-Fundación Eduardo Anitua), Vitoria, Spain

Full list of author information is available at the end of the article
}

osseointegration and has increased the implant secondary stability $[2,3]$. Recently, elements of the extracellular cellular matrix have been introduced to bio-activate the dental implant surface $[4,5]$.

Calcium is one of these elements that has been studied to enhance the osseointegration process [6, 7]. Recently, Favero et al. have compared modifications of an acidetched surface with calcium ions $\left(\mathrm{UnicCa}^{\circ}\right)$ against a surface modified by a nanometer-scale Discrete Crystalline Deposition $\left(\mathrm{DCD}^{\mathrm{mm}}\right)$ of Calcium Phosphate [8]. The patterns of sequential healing have been similar for the two surfaces, although the UnicCa ${ }^{\circ}$ surface showed a statistically significant higher new bone formation at 2 and 4 weeks. Moreover, the osseointegration process of $\mathrm{UnicCa}^{\circ}$ and the 
SLActive ${ }^{\bullet}$ surfaces has been very similar without statistically significant differences [9].

A research is needed to study if these enhancements to the dental implant surface would improve the outcome of dental implants. It has been reported that $40 \%$ of the implant failures occur during the period of osseointegration (early failures) [10]. The presence of low-density bone is a challenging situation to achieve the success of dental implants and requires specific treatment plan and surgical protocol to minimize the risk of implant failure $[11,12]$. The rehabilitation of posterior maxilla with an implant-supported prosthesis could be complicated by the presence of low-density bone [13].

For that, the aim of this study is to evaluate the early survival of UnicCa dental implants placed in transalveolarly augmented maxillary sinus. The null hypothesis of the study is that the UnicCa ${ }^{\oplus}$ surface does not enhance implant survival nor the marginal bone stability. The principal outcome has been the marginal bone stability and as secondary outcome the implant survival.

\section{Methods}

The manuscript was written following STROBE (Strengthening the Reporting of Observational studies in Epidemiology) guidelines. All described data and treatments were obtained from a single dental clinic in Vitoria, Spain. The time period of the study was between December 2014 and April 2016. Patients' records were retrospectively reviewed to identify patients that fulfilled the following inclusion criteria:

- Male and female patients older than 18 years old.

- Transalveolar sinus floor augmentation.

- The insertion of dental implants.

Patients/implants were excluded if not completed with all these criteria. Patients with incomplete data were also excluded. An exemption from IRB approval of the study protocol was granted by the author's institution as it was a retrospective study, and the evaluated medical devise had already been approved for clinical use. This study was performed following the Helsinki declaration regarding the investigation with human subjects.

The principal outcome was the marginal bone loss. The experimental group was composed of the dental implants with $\mathrm{Ca}^{2+}$ ions (UnicCa ${ }^{\circledR}$ surface), and the control group was composed of the implants having the same surface as the UnicCa ${ }^{\oplus}$ but without the calcium ion modification (known as Optima ${ }^{\circ}$ surface). The surface is acid-etched to generate a multi-scale roughness at the different parts of the implant (neck, valleys, and threads) in adaptation to the different biological needs: homogenous and attenuated roughness at the neck to avoid the risk of bacterial colonization, micro-roughness at the valleys to enhance the osseointegration, and micro-roughness + pores at the threads to enhance anchorage.

\section{Outcome assessment}

Data about patients' age and sex were collected. Cone-beam CT scans were visualized in BTI Scan III (Biotechnology Institute, Vitoria, Spain) to measure the residual bone height and the bone density at the surgical site. The sequence of bone drilling was determined according to the bone density [14].

Implant survival determined whether the implant was still physically in the mouth or lost at the time of evaluation. To assess the marginal bone stability, the distance between the uppermost point of the implant platform and the most coronal bone-implant contact was measured mesial and distal to the implant by a computer software (Sidexis, Sirona, USA). Implant length was used to calibrate the linear measurements on the radiograph.

\section{Surgical procedure}

The plasma rich in growth factors (PRGF) was prepared using the Endoret ${ }^{\oplus}$ system following the manufacturer instructions (BTI Biotechnology Institute, Vitoria, Spain). The technique for transalveolar sinus floor elevation is explained elsewhere [15]. Briefly, conventional drills working at low speed $(150 \mathrm{rpm})$ without irrigation was used to prepare the implant site. A frontal cutting drill was then introduced to prepare the last $1 \mathrm{~mm}$ of the implant alveolus. When a window (half of the sinus floor) was created, a well-retracted fibrin plug was introduced. The sinus floor could be opened further, if it was needed. A blunt hand instrument was introduced to push apically the fibrin membrane and to elevate the Schneiderian membrane, simultaneously. The area below the Schneiderian membrane was grafted by PRGF clot. Before implant insertion, the implant socket was irrigated with PRGF. The implants were inserted by a surgical motor at a torque value of $25 \mathrm{~N} \mathrm{~cm}$. Then, the implant was completed seated with a calibrated torque wrench.

After completing the surgical and prosthetic phases, the patient was reviewed at 6 and 12 months during the observation period of the study.

\section{Statistical analysis}

Data collection and analysis were performed by an independent examiner (other than restorative dentist and surgeon). A descriptive analysis of the implant location, length, diameter, bone grafting, and marginal bone loss was performed by considering the implant as the statistical unit of analysis. Shapiro-Wilk test was selected as normality test. Mann-Whitney test was applied to compare the follow-up time, insertion torque, and proximal bone loss between the study groups. Patients' age, sex, and medical history were also analyzed. The 
bone type was compared with Fischer's exact test and the number of implant failures by $x^{2}$ test.

The statistical significance level was 5\% $(p<0.05)$. SPSS v15.0 for Windows statistical software package (SPSS Inc., Chicago, IL, USA) was used.

\section{Results and discussion}

In this study, 51 patients participated with 65 dental implants. The mean age of the patients was $58 \pm 11$ years (range 38 to 72 years) at the time of surgery, and 28 were females.

The experimental group had $34 \mathrm{Ca}^{2+}$-modified dental implants, and the control group had 31 dental implants (without surface modification with calcium ions).

Tables 1 and 2 show the diameters and lengths of the placed dental implants in the experimental and control groups, respectively. Figure 1 shows the anatomical position of the dental implants in the study groups. The residual alveolar bone was of type II (12 implants), type III (16 implants), and type IV (6 implants) in the experimental group. Table 3 shows the bone type in the control group that had significantly more bone of better quality. Dental implants were placed at a mean insertion torque $>30 \mathrm{~N} \mathrm{~cm}$ in both groups (Table 3). The healing time was 4 months. They were mainly supporting fixed screw-retained prostheses, and delayed implant loading was performed.

No intraoperative complications were recorded. During the follow-up period (13 months), no implant failure was encountered in the experimental group. The control group had two implant failures. However, these differences were not statistically significant (Table 3). The mesial and distal bone loss in the experimental group was $0.3 \pm 0.5$ and $0.5 \pm 7 \mathrm{~mm}$, respectively. The proximal bone loss was significantly lower in the experimental group (Table 3 ).

The results of this study do not support the acceptance of the null hypothesis. The modification of an acid-etched surface with calcium ions $\left(\mathrm{UnicCa}^{\circ}\right)$ has enhanced the marginal bone stability.

Maxillary sinus floor elevation using the transalveolar approach may be a valid and less invasive supplement to the lateral window technique $[16,17]$. A prerequisite for using this technique is that primary implant stability could be achieved. Implant's primary stability is the result of quantity and quality of hosting bone, the design

Table 1 Length and diameter of the dental implants in the experimental group

\begin{tabular}{llllllll}
\hline & & \multicolumn{3}{l}{ Diameter $(\mathrm{mm})$} & Total \\
\cline { 3 - 6 } & & 4.25 & 5.00 & 5.50 & 6.00 & 6.25 & \\
\hline Length & 5.5 & 1 & 3 & 3 & 0 & 0 & 7 \\
(mm) & 6.5 & 0 & 5 & 12 & 4 & 2 & 23 \\
& 7.5 & 0 & 2 & 1 & 0 & 1 & 4 \\
Total & & 1 & 10 & 16 & 4 & 3 & 34 \\
\hline
\end{tabular}

Table 2 Length and diameter of the dental implants in the control group

\begin{tabular}{llllllll}
\hline & \multicolumn{9}{l}{ Diameter $(\mathrm{mm})$} & Total \\
\cline { 3 - 6 } & & 4.25 & 5.00 & 5.50 & 6.00 & 6.25 & \\
\hline Length & 5.5 & 0 & 1 & 1 & 0 & 0 & 2 \\
$(\mathrm{~mm})$ & 6.5 & 0 & 2 & 6 & 0 & 4 & 12 \\
& 7.5 & 0 & 1 & 10 & 1 & 2 & 14 \\
& 8.5 & 0 & 0 & 2 & 1 & 0 & 3 \\
Total & & 0 & 4 & 19 & 2 & 6 & 31 \\
\hline
\end{tabular}

of the implant, and the drilling technique [18]. Implant macro-design is a parameter that significantly influences implant primary stability. $\mathrm{Ca}^{2+}$-modified dental implants were placed following the same surgical procedure described by Anitua et al. [15] to place the same dental implant but without $\mathrm{Ca}^{2+}$. For that, no statistically significant differences in primary stability were found between the two dental implants.

Unlike $\mathrm{Ca}^{2+}$-modified dental implants, two early implant losses were observed for the same dental implants but without $\mathrm{Ca}^{2+}$. Moderately rough implant surface has enhanced implant osseointegration and has increased the implant secondary stability [2, 3, 19]. Hydrophilic moderately rough surfaces showed faster osseointegration compared to those with hydrophobic characteristics $[20,21] . \mathrm{Ca}^{2+}$ ions have been shown to protect the hydrophilic implant surface against aging and the formation of carbon-rich species $[4,6]$.

Upon exposure to blood plasma, $\mathrm{Ca}^{2+}$-modified surface has induced surface clot formation, platelet adsorption, and activation [6]. By using a peri-implant gap model in

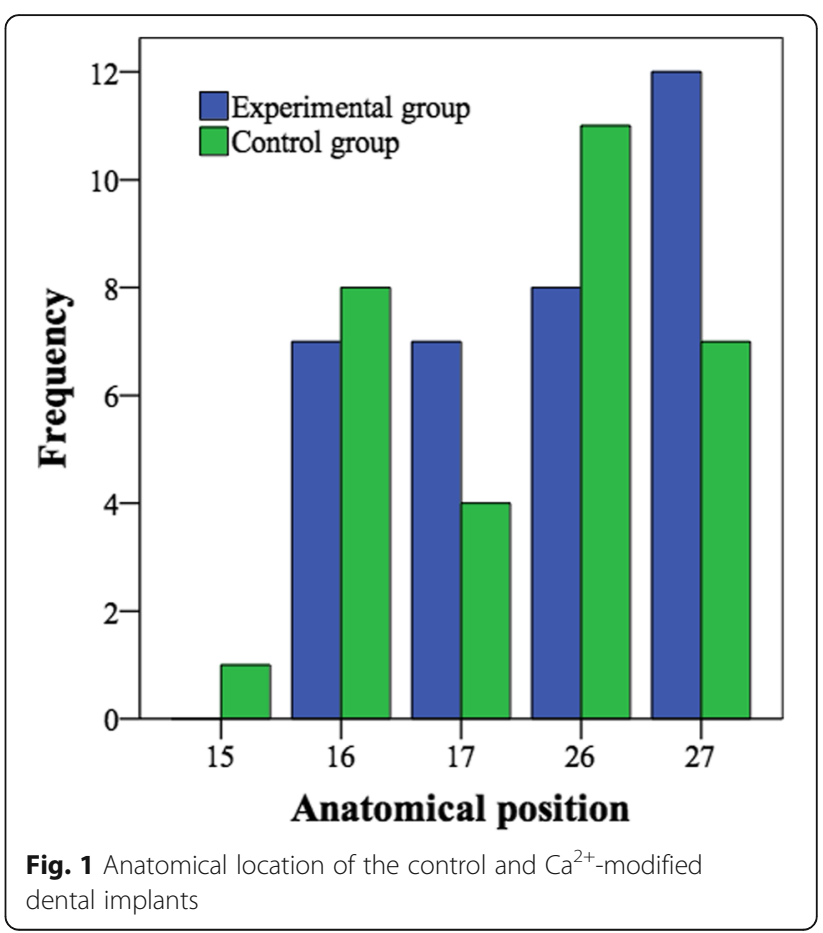


Table 3 Outcomes of experimental and control groups

\begin{tabular}{|c|c|c|c|c|}
\hline \multirow{2}{*}{\multicolumn{2}{|c|}{$\begin{array}{l}\text { Variable } \\
\text { Number of implants }\end{array}$}} & \multirow{3}{*}{$\begin{array}{l}\text { Experimental } \\
34 \\
35.3 \%\end{array}$} & \multirow{3}{*}{$\begin{array}{l}\text { Control } \\
31 \\
74.2 \%\end{array}$} & \multirow{3}{*}{$\begin{array}{l}P<0.05 \\
Y^{a}{ }^{a}\end{array}$} \\
\hline & & & & \\
\hline \multirow[t]{3}{*}{ Bone type } & $\|$ & & & \\
\hline & III & $47.1 \%$ & $22.6 \%$ & \\
\hline & IV & $17.6 \%$ & $3.2 \%$ & \\
\hline \multicolumn{2}{|c|}{ Follow-up time (months) } & $13 \pm 1^{d}$ & $13 \pm 2^{d}$ & $\mathrm{No}^{\mathrm{b}}$ \\
\hline \multicolumn{2}{|c|}{ Insertion torque $(\mathrm{N} \mathrm{cm})$} & $36 \pm 15^{d}$ & $30 \pm 15^{d}$ & $\mathrm{No}^{\mathrm{b}}$ \\
\hline \multicolumn{2}{|c|}{ Implant failure } & 0 & 2 & $\mathrm{No}^{c}$ \\
\hline \multicolumn{2}{|c|}{ Proximal bone loss (mm) } & $0.36 \pm 0.42^{d}$ & $0.61 \pm 0.39^{d}$ & $Y_{e s}^{b}$ \\
\hline
\end{tabular}

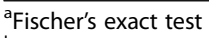

${ }^{b}$ Mann-Whitney test

${ }^{c} x^{2}$ test

${ }^{\mathrm{d}}$ Mean \pm standard deviation

rabbit, $\mathrm{Ca}^{2+}$-modified surface has significantly improved peri-implant bone volume and density at 2 weeks and bone-to-implant contact at 8 weeks [6]. $\mathrm{Ca}^{2+}$-modified surface presented a significantly more new bone formation at 2 and 4 weeks compared to a surface modified by nanometer-scale discrete crystalline deposition of calcium phosphate [8].

In this study, the modifications of an acid-etched surface with calcium ions have significantly decreased the marginal bone loss. Preservation of the crestal bone has been higher in $\mathrm{Ca}^{2+}$-modified implants compared to unmodified implants. One of the criteria of dental implant success as defined by Buser et al. [22] and modified by Albrektsson et al. [23] is the absence of persistent peri-implant bone resorption greater than $1.5 \mathrm{~mm}$ during the first year of loading and $0.2 \mathrm{~mm}$ per year during the following years. Östman et al. have documented the outcomes of dental implants modified with nanometer-scale discrete crystalline deposition of calcium phosphate [24]. The dental implants have been immediately loaded by the fixed prostheses in both maxillary and mandibular regions. The average marginal bone resorption was 0.37 $\pm 0.39 \mathrm{~mm}$ during the first year in function. This outcome might be related to the implant surface modification.

This study was limited by the retrospective design, data dependency on the accuracy of the patients' record, and the short follow-up. Further prospective controlled studies with a long-term follow-up are required. The use of panoramic radiographs could be a source of error in measurement that was reduced by performing a $1: 1$ calibration of the radiograph. This would render the measurements sufficiently accurate for clinical use [25].

\section{Conclusions}

The modification of an acid-etched surface with calcium ions $\left(\mathrm{UnicCa}^{\circ}\right.$ ) seems to enhance the marginal bone stability of dental implants, placed after transalveolar sinus floor elevation.

\section{Abbreviations}

PRGF: Plasma rich in growth factors; STROBE: Strengthening the Reporting of Observational studies in Epidemiology

\section{Acknowledgements}

Not applicable

Funding

No funding was received for this study.

Availability of data and materials

The data will not be shared but are available upon request.

Authors' contributions

$E A, L P$, and MHA was responsible for the concept/design of the study. EA, $L P$, and MHA collected the data. LP and MHA interpreted and analyzed the data. LP and MHA drafted the article. EA, LP, and MHA critically revised and aproved the article. All authors read and approved the final manuscript.

\section{Ethics approval and consent to participate}

An exemption from IRB approval of the study protocol was granted by the author's institution as it was a retrospective study, and the evaluated medical device had already been approved for clinical use. This study was performed following the Helsinki declaration regarding the investigation with human subjects.

\section{Consent for publication}

Not applicable.

\section{Competing interests}

Eduardo Anitua is the Scientific Director of BTI Biotechnology Institute (Vitoria, Spain). He is the head of the Foundation Eduardo Anitua, Vitoria, Spain. Laura Piñas has no conflict of interest. Mohammad Hamdan Alkhraisat is a scientist at BTI Biotechnology Institute (Vitoria, Spain).

\section{Publisher's Note}

Springer Nature remains neutral with regard to jurisdictional claims in published maps and institutional affiliations.

\section{Author details}

${ }^{1}$ Private practice in oral implantology, Clínica Eduardo Anitua, Vitoria, Spain. ${ }^{2}$ University Institute for Regenerative Medicine and Oral Implantology - UIRMI (UPV/EHU-Fundación Eduardo Anitua), Vitoria, Spain. ${ }^{3}$ BTI Biotechnology Institute, Vitoria, Spain. ${ }^{4}$ Universidad Europea de Madrid, Madrid, Spain. ${ }^{5}$ Eduardo Anitua Foundation, C/Jose Maria Cagigal 19, 01007 Vitoria, Spain.

Received: 4 September 2017 Accepted: 15 November 2017 Published online: 04 December 2017

\section{References}

1. Moraschini V, Poubel LA, Ferreira VF, Barboza Edos S. Evaluation of survival and success rates of dental implants reported in longitudinal studies with a follow-up period of at least 10 years: a systematic review. Int J Oral Maxillofac Surg. 2015:44:377-88.

2. Berglundh T, Abrahamsson I, Lang NP, Lindhe J. De novo alveolar bone formation adjacent to endosseous implants. Clin Oral Implants Res. 2003;14: 251-62.

3. Buser D, Schenk RK, Steinemann S, Fiorellini JP, Fox CH, Stich $H$. Influence of surface characteristics on bone integration of titanium implants. A histomorphometric study in miniature pigs. J Biomed Mater Res. 1991;25:889-902.

4. Anitua E, Tejero R, Alkhraisat MH, Orive G. Platelet-rich plasma to improve the bio-functionality of biomaterials. BioDrugs. 2013;27:97-111.

5. Tejero R, Anitua E, Orive G. Toward the biomimetic implant surface: biopolymers on titanium-based implants for bone regeneration. Prog Polym Sci. 2014;39:1406-47.

6. Anitua E, Prado R, Orive G, Tejero R. Effects of calcium-modified titanium implant surfaces on platelet activation, clot formation, and osseointegration. J Biomed Mater Res A. 2015;103:969-80. 
7. Mendes VC, Moineddin R, Davies JE. The effect of discrete calcium phosphate nanocrystals on bone-bonding to titanium surfaces. Biomaterials. 2007;28:4748-55.

8. Favero R, Botticelli D, Antunes AA, Martinez Sanchez R, Caroprese M, Salata LA. Sequential healing at calcium- versus calcium phosphate-modified titanium implant surfaces: an experimental study in dogs. Clin Implant Dent Relat Res. 2016;18:369-78.

9. Favero R, Lang NP, Salata LA, Neto EC, Caroprese M, Botticelli D. Sequential healing events of osseointegration at UnicC ${ }^{\oplus}$ and SLActive ${ }^{\circledast}$ implant surfaces: an experimental study in the dog. Clin Oral Implants Res. 2016;27:203-10.

10. Esposito M, Hirsch JM, Lekholm U, Thomsen P. Biological factors contributing to failures of osseointegrated oral implants. (II). Etiopathogenesis. Eur J Oral Sci. 1998;106:721-64.

11. Bahat O. Branemark system implants in the posterior maxilla: clinical study of 660 implants followed for 5 to 12 years. Int J Oral Maxillofac Implants. 2000;15:646-53.

12. Friberg B, Sennerby L, Meredith N, Lekholm U. A comparison between cutting torque and resonance frequency measurements of maxillary implants. A 20month clinical study. Int J Oral Maxillofac Surg. 1999;28:297-303.

13. Jaffin RA, Berman CL. The excessive loss of Branemark fixtures in type IV bone: a 5-year analysis. J Periodontol. 1991;62:2-4.

14. Anitua E, Alkhraisat MH, Pinas L, Orive G. Efficacy of biologically guided implant site preparation to obtain adequate primary implant stability. Ann Anat. 2015;199:9-15.

15. Anitua E, Alkhraist MH, Piñas L, Orive G. Association of transalveolar sinus floor elevation, platelet rich plasma, and short implants for the treatment of atrophied posterior maxilla. Clin Oral Implants Res. 2015;26:69-75.

16. Del Fabbro M, Corbella S, Weinstein T, Ceresoli V, Taschieri S. Implant survival rates after osteotome-mediated maxillary sinus augmentation: a systematic review. Clin Implant Dent Relat Res. 2012;14(Suppl 1):e159-68.

17. Peleg M, Garg AK, Mazor Z. Predictability of simultaneous implant placement in the severely atrophic posterior maxilla: a 9-year longitudinal experience study of 2132 implants placed into 731 human sinus grafts. Int J Oral Maxillofac Implants. 2006;21:94-102

18. Rabel A, Kohler SG, Schmidt-Westhausen AM. Clinical study on the primary stability of two dental implant systems with resonance frequency analysis. Clin Oral Investig. 2007;11:257-65.

19. Wennerberg A, Albrektsson T, Andersson B, Krol JJ. A histomorphometric and removal torque study of screw-shaped titanium implants with three different surface topographies. Clin Oral Implants Res. 1995;6:24-30.

20. Bosshardt DD, Salvi GE, Huynh-Ba G, Ivanovski S, Donos N, Lang NP. The role of bone debris in early healing adjacent to hydrophilic and hydrophobic implant surfaces in man. Clin Oral Implants Res. 2011;22:357-64.

21. Lang NP, Salvi GE, Huynh-Ba G, Ivanovski S, Donos N, Bosshardt DD. Early osseointegration to hydrophilic and hydrophobic implant surfaces in humans. Clin Oral Implants Res. 2011:22:349-56.

22. Buser D, Weber HP, Bragger U, Balsiger C. Tissue integration of one-stage implants: three-year results of a prospective longitudinal study with hollow cylinder and hollow screw implants. Quintessence Int. 1994;25:679-86.

23. Albrektsson T, Zarb GA. Determinants of correct clinical reporting. Int J Prosthodont. 1998;11:517-21.

24. Ostman PO, Wennerberg A, Albrektsson T. Immediate occlusal loading of NanoTite PREVAIL implants: a prospective 1-year clinical and radiographic study. Clin Implant Dent Relat Res. 2010;12:39-47.

25. Schulze R, Krummenauer F, Schalldach F, d'Hoedt B. Precision and accuracy of measurements in digital panoramic radiography. Dentomaxillofac Radiol. 2000;29:52-6.

\section{Submit your manuscript to a SpringerOpen ${ }^{\circ}$ journal and benefit from:}

- Convenient online submission

- Rigorous peer review

- Open access: articles freely available online

- High visibility within the field

- Retaining the copyright to your article

Submit your next manuscript at $>$ springeropen.com 